\title{
AUSTRIAN TRANSITION TO OPEN ACCESS (AT2OA)
}

\section{von Bruno Bauer, Andreas Ferus, Lothar Hölbling und Tobias Zarka}

Zusammenfassung: Die aktuelle Ausgabe der „Mitteilungen der Vereinigung Österreichischer Bibliothekarinnen und Bibliothekare" (Mitteilungen der VÖB) ist dem Schwerpunktthema Austrian Transition to Open Access, einem Hochschulraumstrukturmittelprojekt mit der Laufzeit 2017 bis 2020 gewidmet, an dem alle 21 öffentlichen Universitäten in Österreich beteiligt sind. Die Schwerpunktausgabe beinhaltet einen Übersichtsbeitrag zu Projekt, Beiträge zu den vier Teilprojekten sowie einen Überblick über die vom Bundesministerium für Wissenschaft geförderten Hochschulraumstrukturmittelprojekte (2014-2020), an denen Universitätsbibliotheken der öffentlichen Universitäten beteiligt waren bzw. sind.

Schlagwörter: Österreich; öffentliche Universität; Universitätsbibliothek; Hochschulraumstrukturmittelprojekt; Austrian Transition to Open Access; AT2OA; Open Access; Editorial

\section{AUSTRIAN TRANSITION TO OPEN ACCESS (AT2OA)}

Abstract: The current issue of the "Communications of the Association of Austrian Librarians" (Mitteilungen der VÖB) is dedicated to the topic Austrian Transition to Open Access, a Higher Education Area Structural Funding project lasting 20172020, in which all 21 Austrian public universities are involved. The special issue includes an overview article on the project, contributions on the four subprojects and an overview of the Higher Education Structural Research projects (2014-2020) cofunded by the Federal Ministry of Science, in which libraries of the public universities have been or are involved.

Keywords: Austria; public university; University Library; Austrian Transition to Open Access; Higher Education Area Structural Funding project; AT2OA; Open Access; Editorial

DOI: https://doi.org/10.31263/voebm.v72i1.2270

(c) Bruno Bauer, Andreas Ferus, Lothar Hölbling, Tobias Zarka

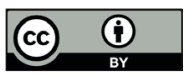

Dieses Werk ist lizenziert unter einer

Creative-Commons-Lizenz Namensnennung 4.0 International 
die vorliegende Ausgabe 1/2019 der Mitteilungen der Vereinigung Österreichischer Bibliothekarinnen und Bibliothekare ist dem Hochschulraumstrukturmittelprojekt „Austrian Transition to Open Access (AT2OA)“ (www.at2oa.at) gewidmet. Open Access war bereits Schwerpunktthema früherer Ausgaben der Mitteilungen der VÖB: „Open Access in Österreich"1 und „Repositorien in Österreich“2.

2016 wurde vom damaligen Bundesministerium für Wissenschaft, Forschung und Wirtschaft im Rahmen der Förderung von Kooperationsprojekten an Hochschulen auch ein Projekt mit der Zielsetzung bewilligt, Open Access in Österreich zu fördern.

Das Hochschulraumstrukturmittelprojekt „Austrian Transition to Open Access (AT2OA)“, an dem alle 21 öffentlichen Universitäten als Kooperationspartnerinnen beteiligt sind und das den Förderzeitraum 2017 bis 2020 umfasst, soll dazu beitragen, Open Access durch geeignete und gemeinsam abgestimmte Maßnahmen voranzubringen. AT2OA, dessen inhaltliche Grundzüge im Rahmen einer vom Forum Universitätsbibliotheken (ubifo) eingesetzten Arbeitsgruppe auf Basis der „Empfehlungen für die Umsetzung von Open Access in Österreich" ${ }^{\text {"3 }}$ erstellt worden sind ${ }^{4}$, gliedert sich in vier Teilprojekte:

In einer "Analyse der Auswirkungen einer Umstellung auf Open Access" werden die finanziellen Auswirkungen einer vollständigen Transformation des Publikationswesens von Closed Access zu Open Access für die am Projekt beteiligten Einrichtungen erhoben [Teilprojekt 1].

Mit der „Finanzierung von Open Access-Übergangsmodellen“ wird die Erweiterung von subskriptionsbasierten Lizenzverträgen um eine Open AccessKomponente (hybrides Open Access) für die Forschenden der am Projekt beteiligten Einrichtungen ermöglicht [Teilprojekt 2].

Maßnahmen für „Auf-, Ausbau und Finanzierung von OA-Publikationsfonds“ sollen die Nutzung des Goldenen Weges zu Open Access für Forschende der am Projekt beteiligten Einrichtungen ermöglichen [Teilprojekt 3].

Mit der „Förderung von OA-Publikationen und alternativen OA-Publikationsmodellen" sollen weitere Aspekte von Open Access ins Blickfeld gerückt werden, die für dessen Weiterentwicklung von essentieller Bedeutung sind, die aber zumeist im Hinblick auf ohnedies knappe Ressourcen vernachlässigt werden [Teilprojekt 4].

Einen sehr guten Beleg für den großen Stellenwert, der Open Access an den Universitäten mittlerweile beizumessen ist, liefert der alle drei Jahre vom Bundesministerium für Bildung, Wissenschaft und Forschung dem Nationalrat gemäß $\S 11$ Universitätsgesetz 2002, BGBI. I Nr. 120/2002, vorzulegende Universitätsbericht. 
Im „Universitätsbericht 2017"5 findet sich im Abschnitt 2.1.3 das Thema „Hochschulraum-Strukturmittel: Ausschreibungen zur Stärkung von Lehre, Forschung und Verwaltungsinnovation", in dem ausgeführt wird, dass die für Verwaltungsinnovation verfügbaren 12,5 Millionen Euro primär für das gemeinsame Vorhaben aller 21 Universitäten (unter Einbindung der Universität für Weiterbildung Krems) zur Vereinheitlichung der Standards in den universitären Kosten- und Leistungsrechnungen während der Implementierungsphase sowie für AT2OA eingesetzt werden: „Mit dem [...] Projekt sollen im Bereich Open Access die universitären Rahmenbedingungen dafür geschaffen werden, dass öffentlich finanzierte Forschungsergebnisse und wissenschaftliche Publikationen schrittweise kostenlos zugänglich gemacht werden." (Universitätsbericht 2017, S. 63)

Auch im Abschnitt 4.5.2 wird das Hochschulraumstrukturmittelprojekt AT2OA beim Thema „Zugänglichkeit von Forschungsleistungen - Open Access an Universitäten“ kurz dargestellt (Universitätsbericht 2017, S. 148-149).

Die Grundzüge des Hochschulraumstrukturmittelprojektes „Austrian Transition to Open Access"sowie erste Zwischenergebnisse wurden in Vorträgen im Rahmen des OANA-Netzwerktreffens $2016^{6}$ in Wien sowie der Open Access-Tage 2016 ${ }^{7}$ in München bzw. $2018^{8}$ in Graz vorgestellt. Ein erster umfassender Projektbericht wurde 2018 in Bibliothek Forschung und Praxis $^{9}$ veröffentlicht. In der aktuellen Schwerpunktausgabe der Mitteilungen der VÖB werden zur Projekthalbzeit erstmals wichtige Ergebnisse aus den einzelnen Teilprojekten publiziert.

\section{Schwerpunktthema}

Brigitte Kromp, Maria Seissl und Tobias Zarka stellen in ihrem Beitrag "Austrian Transition to Open Access (AT2OA) - ein Überblick" das aus Hochschulraumstrukturmitteln geförderte Projekt mit seinen inhaltlichen Schwerpunkten sowie seiner Projekt- und Kommunikationsstruktur kurz vor und schließen nach der Hälfte der Projektlaufzeit mit einer positiven Zwischenbilanz [S. 28-34].

Georg Fessler fasst unter dem Titel „Ausbau von Open Access an den österreichischen Universitäten: Budgetärer Mehrbedarffür die Jahre 2019-2021“ die Ergebnisse der HRSM AT2OA Transition-Studie zusammen, die im Rahmen einer Arbeitsgruppe in Teilprojekt 1 entstanden ist. Der Beitrag behandelt Budgetund Kostenfragen im Zusammenhang mit einem weiteren Ausbau von Open Access an den österreichischen Universitäten. Für die entsprechenden Berechnungen wurde auch der wissenschaftliche Publikationsoutput Österreichs 
für 2016 erhoben und analysiert. Berücksichtigt wurden auch die Open Access-Ausgaben des FWF als wichtigem Fördergeber sowie die mögliche Weiterentwicklung von Konsortialverträgen mit Open Access-Komponenten sowie der Bedarffür den Auf- und Ausbau von Open Access-Publikationsfonds in unterschiedlichen Ausbaustufen [S. 35-49]. Die umfassende Studie, die dem Beitrag von Fessler zugrunde liegt, findet sich auf Zenodo. ${ }^{10}$

Unter dem Titel „Datenerhebung und Analyse des Publikationsoutputs von Forschenden an österreichischen Universitäten und Forschungseinrichtungen 2015 bis 2017 im Rahmen von AT2OA - Werkstattbericht zu einer bibliometrischen Studie" gibt Lothar Hölbling einen kurzen Überblick zu Zielen, Methoden und der Umsetzung des Konzeptes und stellt ausgewählte Ergebnisse aus dem Publikationsoutput-Dataset für das Jahr 2016 vor. Die ermittelten Daten werden für die Analyse der Auswirkung einer Umstellung auf Open Access genutzt, die im Rahmen des Hochschulraumstrukturmittelprojektes „Austrian Transition to Open Access (AT2OA)“ durchgeführt wird [S. 50-58].

„An Austrian Proposal for the Classification of Open Access Tuples (COAT) - Distinguish Different Open Access Types beyond Colors" - unter diesem Titel stellt Patrick Danowski ein Konzept vor, mit dem die unterschiedlichen Varianten von Open Access exakt klassifiziert werden können. Zumeist werden Open Access-Publikationen unterschieden in Gold Open Access (Erstpublikation) und Green Open Access (Zweitpublikation), ergänzt um die Kategorien Hybrid Gold Open Access und neuerdings auch Bronce Open Access. Im Rahmen einer Arbeitsgruppe in Teilprojekt 1 von AT2OA wurde ein Klassifikationsschema erstellt, mit dem anhand von fünf Kriterien - Speicherort, Lizenz, Version, Embargofrist, finanzielle Bedingungen der Publikation - eine exakte Beschreibung der verschiedenen bestehenden, aber auch von sich gegebenenfalls noch entwickelnden Arten von Open Access möglich wird [S. 59-65].

Brigitte Kromp und Frank Koren-Wilhelmer beschreiben unter dem Titel „Finanzierung von Open Access-Übergangsmodellen: Leitlinie für Verlagsverträge für die Open Access Transformation" unter welchen Voraussetzungen ein fairer und dem Open Access-Gedanken angemessener Einsatz finanzieller Mittel für Lizenzverträge mit Open Access-Komponente möglich ist. Im Beitrag werden die drei Transitionsmodelle erläutert, die in der gegenwärtigen Übergangsperiode von einem subskriptions- und lizenzbasierten auf ein Open Access-basiertes Publikationssystem im Rahmen der Kooperation E-Medien Österreich (KEMÖ) zum Einsatz kommen (Offsetting-Deals, Read-and-Publish-Deals, Switches). Vorgestellt wird dabei insbesondere die „Leitlinie für Verlagsverträge für die OA-Transformation im 
Rahmen von HRSM-AT2OA“, die von einer Arbeitsgruppe in Teilprojekt 2 erstellt wurde. Auf Basis dieser Handreichung wurde auch die Entscheidung getroffen, einen Lizenzvertrag mit Transitionskomponente mit dem Verlag Wiley für die Jahre 2018 bis 2020 abzuschließen [S. 66-73].

„Förderbedingungen für Publikationsfonds: Open Access - ganz oder gar nicht" - unter diesem zugespitzten Titel stellen Christof Capellaro und Christian Kaier, aufbauend auf einem von einer Arbeitsgruppe in Teilprojekt 3 erstellten Dokument mit dem Titel „Open-Access-Publikationsfonds. Einrichtung und Förderbedingungen", das derzeit finalisiert und in den nächsten Wochen auf Zenodo bereitgestellt werden wird, Überlegungen zu verschiedenen Aspekten im Zusammenhang mit der Festlegung von Förderbedingungen für Open Access-Publikationsfonds dar. Thematisiert werden die Spannungsfelder, die sich aus disziplinspezifischen Unterschieden, die Frage der Bevorzugung bestimmter Personengruppen in den Förderbedingungen, Vorgaben für Lizenzen und Publikationstypen sowie die grundsätzliche Frage, welche Arten von Ausgaben und welche Open-Access-Finanzierungsmodelle für eine Förderung aus den Mitteln eines Publikationsfonds generell in Betracht kommen [S. 74-88].

„Die Förderung von alternativen, nichtkommerziellen Open Science-Infrastrukturen o-services (OSIS) durch Forschungseinrichtungen in Österreich - Empfehlungen, Kriterien \& Modelle" behandeln Andreas Ferus und Falk Reckling in ihrem Beitrag, der im Kontext einer Arbeitsgruppe in Teilprojekt 4 entstanden ist. Vorgestellt werden Kriterien für die Evaluierung von Open Science-Infrastrukturen \& -Services sowie mögliche Finanzierungsmodelle für deren längerfristige Förderung. Ergänzt wird der Beitrag um eine Matrix, in der die bereits von mehreren oder einzelnen Institutionen in Österreich unterstützten Open Science-Infrastrukturen \& -services sowie die angewendeten Fördermodelle übersichtlich zusammengestellt sind [S. 89-105]. Auch der Beitrag von Ferus und Reckling wurde vorab als Preprint in Zenodo eingestellt. ${ }^{11}$

Den Abschluss der Schwerpunktausgabe zu AT2OA bildet ein Beitrag von Bruno Bauer und Maria Seissl über „Kooperationen von Universitätsbibliotheken der öffentlichen Universitäten in Österreich im Rahmen der Hochschulraumstrukturmittelprojekte". Vom für Wissenschaft und Forschung zuständigen Bundesministerium werden seit 2014 Hochschulraumstrukturmittel als Anschubfinanzierung für universitäre Kooperationsvorhaben bereitgestellt. Im Zuge der Ausschreibungen von 2013 bzw. 2017 für Projekte der Verwaltung wurden auch alle Projekte, deren Konzeption auf Initiative des Forums Universitätsbibliotheken Österreichs erfolgt ist, vom Bundesministerium als förderungswürdig ausgewählt. Im Beitrag werden die teils schon abgeschlossenen, teils noch laufenden Projekte sowie deren Ergebnisse vorgestellt: „e-Infrastructures 
Austria“ (2014-2016), „e-Infrastructures Austria Plus“ (2017-2019), „Entwicklung und Umsetzung eines Beschaffungsprozesses für österreichische Universitätsbibliotheken“ (2014-2016), „Austrian Transition to Open Access“ (2017-2020) und „Open Education Austria“ (2017-2018). Die bisherigen Ergebnisse und die noch umzusetzenden Vorhaben aus den von den Universitätsbibliotheken betriebenen Hochschulraumstrukturmittelprojekten bilden die Basis dafür, dass die Informationsinfrastruktur in Österreich für Forschung und Lehre im europäischen und internationalen Kontext - auch im Hinblick auf die Anforderungen der European Open Science Cloud - weiterentwickelt werden kann. Die Bedeutung der von den Universitätsbibliotheken initiierten und zum Teil gemeinsam mit anderen Serviceeinrichtungen der Universitäten betriebenen Hochschulraumstrukturmittelprojekte findet auch in einem „Vortrag an den Ministerrat" seinen Niederschlag, in dem der für Wissenschaft und Forschung zuständige Bundesminister Heinz Faßmann diese Projekte unter dem Punkt „Aktuelle Strategien, Initiativen und Fördermaßnahmen" anführt und ihnen auch beim Punkt „Strategien und Ziele für die künftigen Entwicklungen" eine wichtige Rolle beimisst ${ }^{12}$ [S. 106-123].

\section{Aus der VÖB}

Werner Schlacher informiert in der Rubrik „Aus Präsidium und Vorstand der $V O ̈ B$ “ über den bevorstehenden 34. Österreichischen Bibliothekartag, der unter dem Motto „Künstliche Intelligenz in Bibliotheken“ von 10. bis 13. September 2019 in Graz stattfinden wird, und gibt eine Vorschau auf den 1. Österreichischen Bibliothekskongress, der von der VÖB und vom Büchereiverband Österreichs (BVÖ) gemeinsam 2021 in Innsbruck veranstaltet wird [S. 17-19].

Mit dem Beitrag „In Memoriam Hermann Riepl (1938-2019)“von Gebhard König beinhaltet die aktuelle Ausgabe der Mitteilungen der VÖB auch einen Nachruf auf den ehemaligen Direktor der Niederösterreichischen Landesbibliothek (1989-1996), der sich für die VÖB als Mitglied des Vorstandes, als Vorsitzender der Kommission für Bibliografie und als Vizepräsident verdient gemacht hat [S. 23-27].

\section{Weitere Beiträge}

Blaženka Klemar Bubić stellt „Die Österreich-Bibliothek in Zagreb (Austrijska Knjiznica Zagreb)“ vor, die 2015 auf Initiative der Österreichischen Bot- 
schaft, des Österreichischen Kulturforums Zagreb und der Abteilung für Germanistik eröffnet worden ist. Mit den seit 1983 auf dem Gebiet der ehemaligen Habsburgermonarchie gegründeten Österreich-Bibliotheken wird das Ziel verfolgt, interessierten Benutzerinnen und Benutzern den Zugang zu österreichischer Literatur, Wissenschaft und zu Informationen aus Österreich zu erleichtern [S. 124-143].

Claudia Sojer interviewt „Privatdozentin Dr. ${ }^{\text {in }}$ Christina Maria Grafinger eine Österreicherin als erste Frau im Vatikan“. Grafinger, 1953 in Vöcklabruck geboren, hat seit 1996 als Bibliothekarin in der Biblioteca Apostolica Vaticana des Vatikanstaates und seit 2009 bis zum Antritt ihres Ruhestandes 2018 als Leiterin deren Archivs gearbeitet. Im Interview schildert sie ihre Zeit in Rom, dessen Highlight im Jahr 2014 ein Besuch bei Papst Benedikt XVI. war [S. 144-154].

Susanne Luger und Marian Miehl informieren über das „ALMA-Forum (ALMA-FachexpertInnen-Forum)“, dessen aktuelle und zukünftige Aufgaben sowie über dessen Zusammensetzung [S. 155-159].

Über das österreichische Netzwerk für Repositorienmanagerlnnen (RepManNet), das im Herbst 2016 unter dem Dach des Forum Universitätsbibliotheken Österreichs (ubifo) eingerichtet wurde, informiert Susanne Blumesberger. In ihrer Mitteilung werden Ziele, Arbeitsgruppen und Kommunikationsinfrastruktur von RepManNet vorgestellt [S. 160-163].

Tereza Kalová schildert in „Mein erster (und zweiter) Einblick in die österreichische Bibliothekslandschaft - Praktika an den Bibliotheken der TU Wien und der MedUni Wien" ihre Erfahrungen, die sie in den beiden Universitätsbibliotheken zwischen Oktober und Dezember 2018 gemacht hat [S. 164-167].

In den „Mitteilungen der OBVSG“ berichtet Mathis Kronschläger über aktuelle Entwicklungen aus dem Österreichischen Bibliothekenverbund [S. 168-172].

Branka Steiner-Ivancevic informiert über die „Eröffnung und Präsentation der digitalisierten Nachlässe von Kurt W. Rothschild und Josef Steindl an der Universitätsbibliothek der WU Wien", die am 29. Oktober 2018 stattgefunden hat [S. 173-176].

Am 21. und 22. November 2018 fand - unmittelbar vor dem Launch der European Open Science Cloud (EOSC) - das „Second EOSCpilot Stakeholders Forum" im Austria Center in Wien statt. Thomas Haselwanter informiert in seinem Bericht über den Ablauf dieser Veranstaltung, die regelmäßig wiederholt werden soll, und über die Zielsetzung des „Stakeholders Forum", das in Form regelmäßiger Treffen an der Weiterentwicklung der EOSC mitwirken soll [S. 177-183].

Sebastian Aigner, Magdalena Andrae, Bruno Bauer, Susanne Blumesberger, Olivia Kaiser und Markus Stumpf berichten unter dem Titel „Kooperativer 
Bericht vom 7. Deutschen Bibliothekskongress: Bibliotheken verändern"vom größten nationalen Bibliothekskongress in Europa, der von 18. bis 21. März 2019 in Leipzig stattgefunden hat und an dem 4.000 Besucherinnen und Besucher, darunter fast 100 aus Österreich, teilgenommen haben [S. 184-213].

In der aktuellen Ausgabe der Mitteilungen der VÖB bringen wir abschließend eine Rezension von Thomas Kuster (Porträtgalerien auf Papier. Sammeln und Ordnen von druckgrafischen Porträts am Beispiel Kaiser Franz' I. von Österreich und anderer fürstlicher Sammler [S. 214-218]).

Mag. Bruno Bauer

ORCID: http://orcid.org/0000-0002-4729-331X Medizinische Universität Wien, Universitätsbibliothek E-Mail: bruno.bauer@meduniwien.ac.at

Mag. Andreas Ferus, MSc ORCID: http://orcid.org/0000-0003-2509-0009 Akademie der bildenden Künste Wien, Universitätsbibliothek E-Mail: a.ferus@akbild.ac.at

Mag. Lothar Hölbling Universität Wien, Bibliotheks- und Archivwesen E-Mail: lothar.hoelbling@univie.ac.at

Tobias Zarka Universität Wien, Bibliotheks- und Archivwesen E-Mail: tobias.zarka@univie.ac.at 
1 Schwerpunktthema „Open Access in Österreich“. In: Mitteilungen der Vereinigung Österreichischer Bibliothekarinnen und Bibliothekare 65 (2012), H. 2. Online unter: https://www.univie.ac.at/voeb/publikationen/voeb-mitteilungen/voeb-mitt-65-2012-2/

2 Schwerpunktthema "Repositorien in Österreich - Status quo und (zukünftige) Herausforderungen“. In: Mitteilungen der Vereinigung Österreichischer Bibliothekarinnen und Bibliothekare 71 (2018), H. 1. https://doi. org/10.31263/voebm.v71i1

3 Bauer, Bruno; Blechl, Guido; Bock, Christoph; Danowski, Patrick; Ferus, Andreas; Graschopf, Anton; König, Thomas; Mayer, Katja; Nentwich, Michael; Reckling, Falk; Rieck, Katharina; Seitz, Peter; Stöger, Herwig; Welzig Elvira: Empfehlungen für die Umsetzung von Open Access in Österreich. In: Mitteilungen der Vereinigung Österreichischer Bibliothekarinnen und Bibliothekare 68 (2015), H. 3/4, S. 580-607. https://doi. org/10.31263/voebm.v68i3.1299

4 Bauer, Bruno; Capellaro, Christof; Ferus, Andreas; Fessler, Georg; Koren, Frank; Kromp, Brigitte; Pörnbacher, Erika; Winkler, Gisela; Cirkovic, Snjezana; Danowski, Patrick; Villányi, Márton: HRSM Projektskizze - Draft für das Projekt „Austrian Transition to Open Access (AT2OA)“. Wien: Forum Universitätsbibliotheken Österreichs (ubifo), 2016.

5 Bundesministerium für Bildung, Forschung und Wissenschaft (Hrsg.): Universitätsbericht 2017. Wien: BMBWF, 2018. Online unter: https:// bmbwf.gv.at/fileadmin/user_upload/wissenschaft/publikationen/Universitätsbericht_2017_barrierefrei_20180312.pdf

6 Bauer, Bruno; Kromp, Brigitte: Hochschulraumstrukturmittel als Katalysator der Open Access Transition (Präsentation). 3. OANA Gesamtveranstaltung, Wien, 01.06.2016. Online unter: https://www.oana.at/ fileadmin/user_upload/k_oana/netzwerktreffen/nr03-2016/Netzwerktreffen_03_OANA_3_Bauer_Kromp_Hochschulraumstrukturmittel_ als_Katalysator_der_Open_Access_Transition.pdf

7 Bauer, Bruno: Die Umsetzung der österreichischen Open-Access-Strategie: „Austrian Transition to Open Access“ (Präsentation). Open Access Tage, München, 10.10.2016. Online unter: https://open-access. net/fileadmin/oat/oat16/Dokumente/OAT16_Bauer.pdf

8 Fessler, Georg: AT2OA Transition-Studie: Kalkulationsmethoden für den Ausbau von Open Access in Österreich in den nächsten Jahren (Präsentation). Open Access Tage, Graz, 26.09.2018. Zenodo. https:// doi.org/10.5281/zenodo.1441248

9 Bauer, Bruno; Capellaro, Christof; Ferus, Andreas; Fessler, Georg; Granat, Renata; Hölbling, Lothar; Kaier, Christian; Koren-Wilhelmer, 
Frank; Kromp, Brigitte; Seissl, Maria; Zarka, Tobias: Austrian Transition to Open Access (AT2OA). In: Bibliothek Forschung und Praxis 42 (2018), H. 3, S. 463-475. https://doi.org/10.1515/bfp-2018-0062

10 Fessler, Georg; Hölbling, Lothar: Ausbau von Open Access an den österreichischen Universitäten: Budgetärer Mehrbedarf für die Jahre 2019-2021. Wien: AT2OA, 2019. Zenodo. http://doi.org/10.5281/ zenodo. 2621015

11 Reckling, Falk; Ferus, Andreas: Die Förderung von alternativen, nicht-kommerziellen Open Science-Infrastrukturen \& -Services (OSIS) durch Forschungseinrichtungen in Österreich - Empfehlungen, Kriterien \& Modelle (Version 1.1). Wien 2019. Zenodo. http://doi.org/10.5281/ zenodo. 2549452

12 Faßmann, Heinz: Vorhaben des Bundesministers für Bildung, Wissenschaft und Forschung zur ZUKUNFT DIGITALISIERUNG - Die österreichischen Universitäten am Weg ins digitale Zeitalter. Vortrag an den Ministerrat, 07.06.2018. Online unter: https://www.bundeskanzleramt. gv.at/documents/131008/877075/21_9_mrv.pdf/1e321 eea-066c4d71-8143-b757d21cfe21 